
PROF. RUBIAO IIEIRA.

\title{
SOBRE A FREQUÊNCIA DOS SINTOMAS GASTRO-INTESTINAIS (')
}

\section{Ddo. HELIO LOURENCO DE OLIVEIRA Acadêmicos RAPHAEL GIANNELLA e FIRMINO CAMPOS}

O material aproveitado para este trabalho é representado pơr 444 observações clínicas do arquivo da 2 a $^{\mathrm{a}}$ M. H. da Santa Casa de Misericórdia, serviço do prof. Rubião Meira. Não houve nenhuma seleção de casos, tomando-se as observações a partir das mais recentes para as mais antigas; apenas foram desprezadas as em que não se encontrou qualquer referência, afirmativa ou negativa, 'sobre a ocorrência de sintomas gastro-intestinais.

Os diferentes sintomas considerados nos quadros abaixo não foram discriminados "a priori", mas colhidos das mesmas observações estudadas. O rigor da sua discriminação se subordinou à precisão das anamneses revistas, refletindo naturalmente, em grande parte, a precisão das informações dos pacientes.

\section{RESULTADOS}

Resultados globais - O quadro I resume a frequência geral dos sintomas, que se apresentam em $7.2 \%$ dos casos. Esse valor se afasta muito do da frequência de moléstias com localisações definidas no tubo gastro-intestinal; estas se representam por, 35 casos de moléstias do esôfago, estômago e duodeno, e 106 do intestino delgado e colons, num total de 141 casos, $31,7 \%$ do total.

Os sintomas mais frequentes, presentes em mais de quarta parte dos casos, são perda de apetite, prisão de ventre e um grupo mais ou menos họmogêneo dos sintomas gástricos (azia, e ardor, plenitude ou peso epigástricos); os dois primeiros são tambem os que mais frequentemente aparecem como sintomas isolados.

. Segundo os tipos morfológicos - A frequência dos sintomas gastro-intestinais em relação com o tipo morfológico (quadro II) foi

(I) Trabalho apresentado no 1.0 Congresso dos Estudantes de Medicina de São Paulo, outubro de 1940. 
estabelecida com base em dados de 284 observações. A definiçầo do tipo morfológico, em todas essas observações, obedece ao critério cla prática clínica, decorrendo da impressão da inspeção geral, da relação entre as dimensōes dos membros e do tronco, do valor do ángulo de Charpy. Os resultados enfeixados no quadro II mostram diferenças entre os números percentuais relativos aos 3 tipos mor-

QuAdro I

OBSERVAÇOES REFERENTES A 444 CASOS

\begin{tabular}{|c|c|c|}
\hline Sem sintomas gastro-intestinais & 125 casos & $28 \%$ \\
\hline 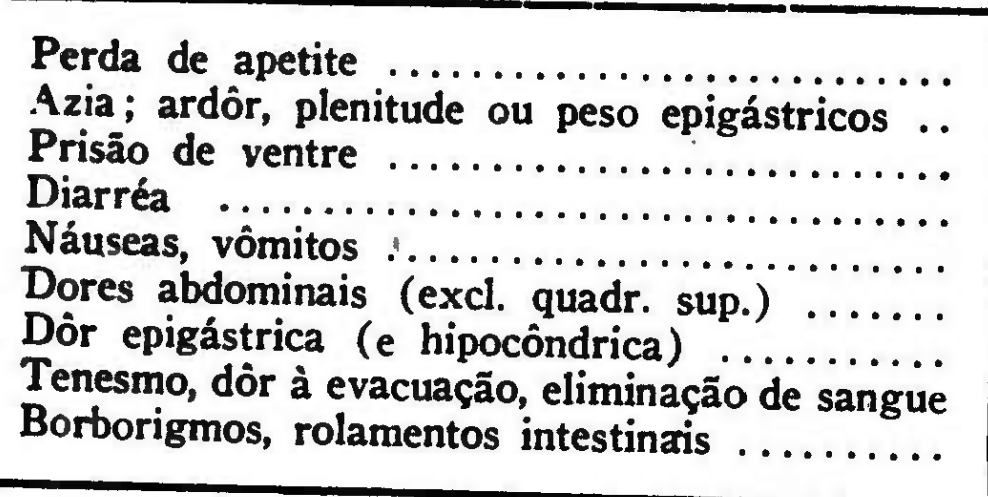 & $\begin{array}{l}115 \text { casos } \\
112 " \\
112 " \\
91 \\
81 " \\
75 " \\
49 " \\
47 " \\
24 "\end{array}$ & $\begin{array}{l}25,4 \% \\
25,2 \% \\
25,2 \% \\
20,4 \% \\
18,2 \% \\
16,8 \% \\
11 \% \\
10,5 \% \\
5,4 \%\end{array}$ \\
\hline 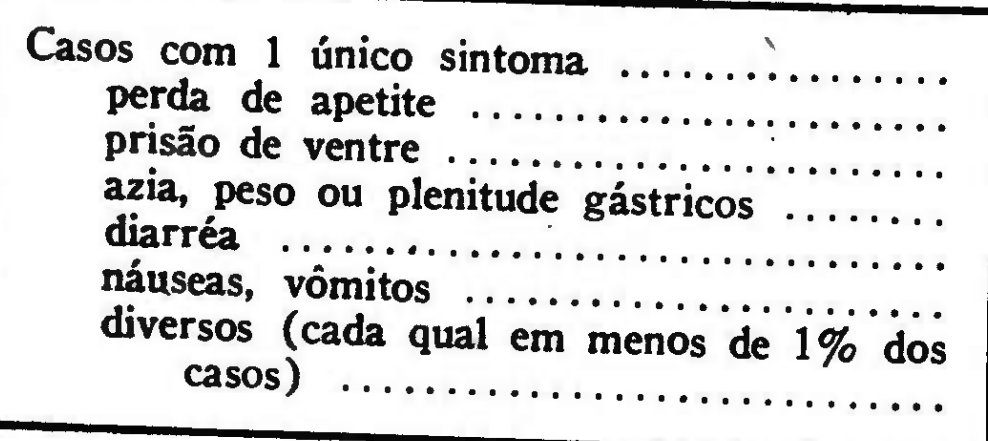 & $\begin{array}{r}92 \\
23 \\
22 \\
12 \\
10 \\
9 \\
16\end{array}$ & $\begin{array}{r}20,7 \% \\
5,2 \% \\
5 \% \\
2,7 \% \\
2,2 \% \\
2 \% \\
3,6 \%\end{array}$ \\
\hline
\end{tabular}

fológicos, referentes a todos os sintomas; essas diferenças não auem que conclusōes, antes de tudo devido ao pequeno valor dos totais equivale contradas quasi 2 por cento). Salientamos, porém, as variações enou peso epigástricos" e aos capo sintomático "azia; ardor, plenitude que se afastam nitidamente casos "sem sintomas gastro-intestinais", quência dos diversos sintomas. Aquem geral das variações de fresentam com frequência menor Aqueles sintomas gástricos se apredestes mesmos é que se encontra a braquitipos, e ainda no grupo sintomas gastro-intestinais.

Segundo a idade - Cónsideramos 3 grupos etários (quadro
III). fazendo o $20^{\circ}$ e o $30^{\circ}$ corresponder da idade adulta. O $10^{\circ}$ grupo, dos indivír a dois períodos distintos se apresenta pouco numeroso, donde rios sintomas calculada para esse grupo frequência relativa dos vápara os 2 outros. 
QUADRO II

OBSERVAÇOES REFERENTES A 284 CASOS

\begin{tabular}{|c|c|c|c|c|c|c|}
\hline Tipo morfológico . & \multicolumn{2}{|c|}{ LoNGITIPOS } & \multicolumn{2}{|c|}{ NORMOTIPOS } & \multicolumn{2}{|c|}{ BRAQUITIPOS } \\
\hline Nimero de casos & \multicolumn{2}{|c|}{94} & \multicolumn{2}{|c|}{132} & \multicolumn{2}{|c|}{58} \\
\hline Sintomas & $\begin{array}{l}\text { n. de } \\
\text { casos }\end{array}$ & $\begin{array}{l}\% \text { do } \\
\text { total }\end{array}$ & $\begin{array}{l}\text { n. de } \\
\text { casos }\end{array}$ & $\begin{array}{c}\% \text { do } \\
\text { total }\end{array}$ & $\begin{array}{l}\text { n. de } \\
\text { casos }\end{array}$ & $\begin{array}{l}\% \text { do } \\
\text { total }\end{array}$ \\
\hline 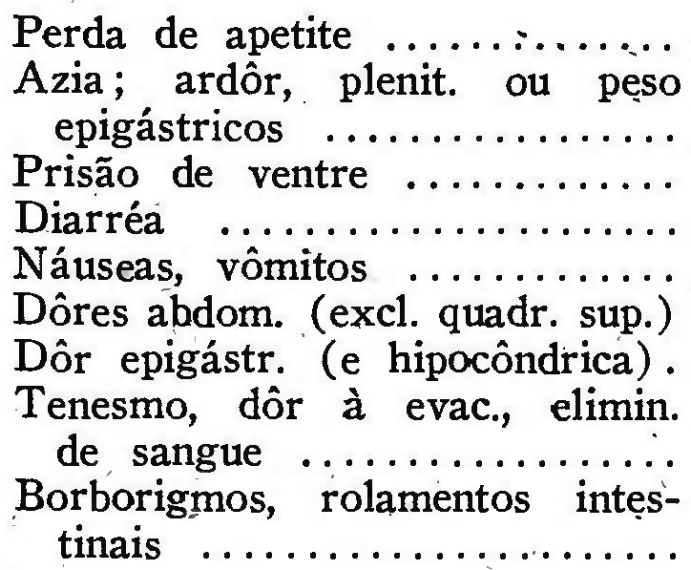 & $\begin{array}{r}25 \\
26 \\
23 \\
19 \\
15 \\
13 \\
7 \\
11 \\
\\
4\end{array}$ & $\begin{array}{l}26,5 \\
27,5 \\
24,5 \\
20 \\
16 \\
14 \\
7,5 \\
12 \\
4\end{array}$ & $\begin{array}{r}36 \\
47 \\
37 \\
29 \\
27 \\
25 \\
17 \\
14 \\
9\end{array}$ & $\begin{array}{l}27 \\
35,5 \\
28 \\
22 \\
20,5 \\
19 \\
13 \\
10,5 \\
7\end{array}$ & $\begin{array}{r}19 \\
8 \\
15 \\
9 \\
10 \\
11 \\
5 \\
7 \\
7 \\
2\end{array}$ & $\begin{array}{l}33 \\
14 \\
25,5 \\
16 \\
17 \\
19 \\
8,5 \\
12 \\
3,5\end{array}$ \\
\hline Sem sintomas g.-i. & 25 & 26,5 & 35 & 26,5 & 21 & 36 \\
\hline
\end{tabular}

QUADRo III

\section{OBSERVAÇŐES REFERENTES A 444 CASOS}

\begin{tabular}{|c|c|c|c|c|c|c|}
\hline Idade $\ldots . . . . . . . . \ldots \ldots \ldots . .$. & \multicolumn{2}{|c|}{10 a 20 anos } & \multicolumn{2}{|c|}{21 a 40 anos } & \multicolumn{2}{|c|}{ m. de 41 anos } \\
\hline Número de casos .. & & 48 & & 24 & & 72 \\
\hline Srntomas & $\begin{array}{l}\text { n. de } \\
\text { casos }\end{array}$ & $\begin{array}{r}\% \text { do } \\
\text { total }\end{array}$ & $\begin{array}{l}\text { n. de } \\
\text { casos }\end{array}$ & $\begin{array}{l}\% \text { do } \\
\text { total }\end{array}$ & $\begin{array}{l}\text { n. de } \\
\text { casos }\end{array}$ & $\begin{array}{l}\% \text { do } \\
\text { total }\end{array}$ \\
\hline 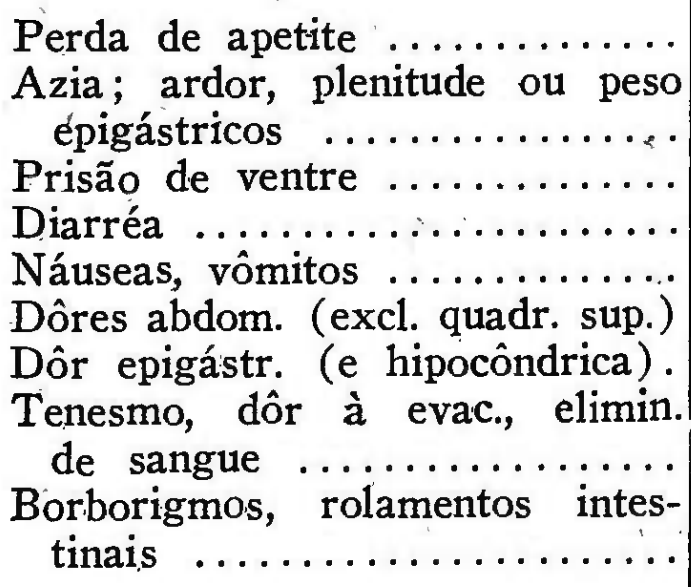 & $\begin{array}{r}15 \\
8 \\
14 \\
1 \\
8 \\
4 \\
6 \\
1 \\
1\end{array}$ & \begin{tabular}{|}
31 \\
16,5 \\
29 \\
2 \\
16,5 \\
8 \\
12,5 \\
2 \\
2
\end{tabular} & $\begin{array}{l}59 \\
65 \\
57 \\
58 \\
52 \\
42 \\
26 \\
24 \\
16\end{array}$ & $\begin{array}{l}26 \\
29 \\
25,5 \\
26 \\
23 \\
19 \\
11,5 \\
11 \\
7\end{array}$ & $\begin{array}{r}41 \\
39 \\
41 \\
32 \\
21 \\
29 \\
17 \\
22 \\
7\end{array}$ & $\begin{array}{l}24 \\
22,5 \\
24 \\
18,5 \\
12 \\
17 \\
10 \\
13 \\
\\
\quad 4\end{array}$ \\
\hline Sem sintomas g.-i. $\ldots \ldots \ldots \ldots$ & 17 & 35,5 & 52 & 23 & 56 & 32,5 \\
\hline
\end{tabular}




\section{QUADRo IV \\ MOLESTIAS DO APARELHO RESPIRATORIO \\ 81 casos}

\begin{tabular}{|c|c|}
\hline Sem sintomas gastro-intestinais $\ldots \ldots \ldots \ldots$ & 28 casos \\
\hline 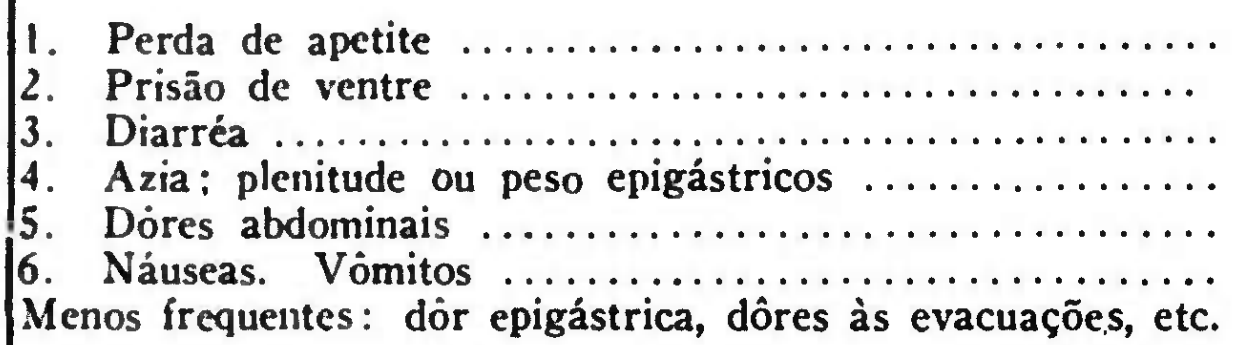 & $\begin{array}{r}24 \text { casos } \\
17 \text { " } \\
15 \text { " } \\
12 \\
8 " ~ \\
7 "\end{array}$ \\
\hline
\end{tabular}

\section{QUADRo IV-a \\ TUBERCULOSE PULMONAR 28 casos}

\begin{tabular}{|c|c|}
\hline Sem sintomas gastro-intestinais $\ldots . \ldots \ldots$ & 12 casos \\
\hline 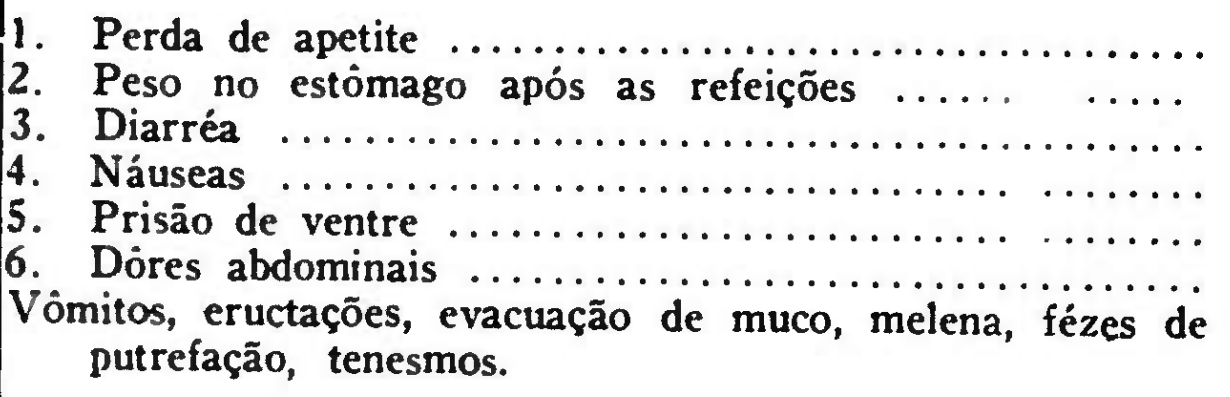 & $\begin{array}{lc}7 & \text { casos } \\
4 & " \\
4 & " \\
3 & " \\
3 & " \\
3 & "\end{array}$ \\
\hline Os sintomas associados de modo variavel. & \\
\hline
\end{tabular}

$$
\text { QUADRo IV-b }
$$

\section{PNEUMONIA LOBAR \\ 18 casos}

\begin{tabular}{|c|c|}
\hline Sem sintomas gastro-intestinais ... & 8 casos \\
\hline 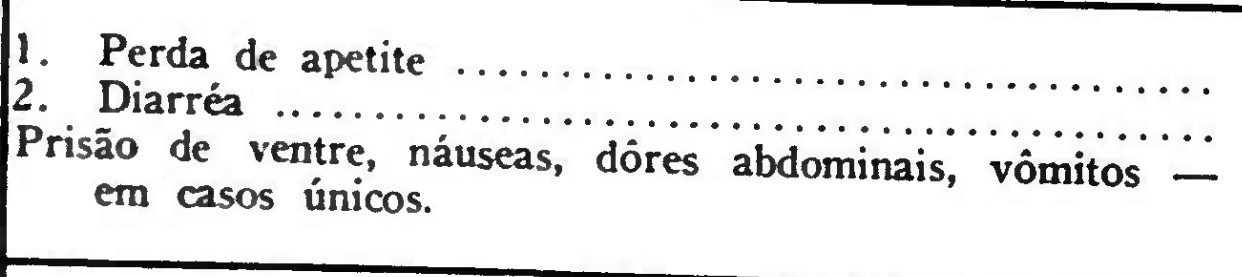 & 5 casos \\
\hline
\end{tabular}




\section{QUAdRo .V}

\section{MOLESTIAS DO APARELHO CIRCULATORIO 105 casos}

\begin{tabular}{|c|c|}
\hline Sèm sintomas gastro-intestinais $\ldots \ldots \ldots \ldots \ldots \ldots \ldots \ldots$ & 34 casos \\
\hline 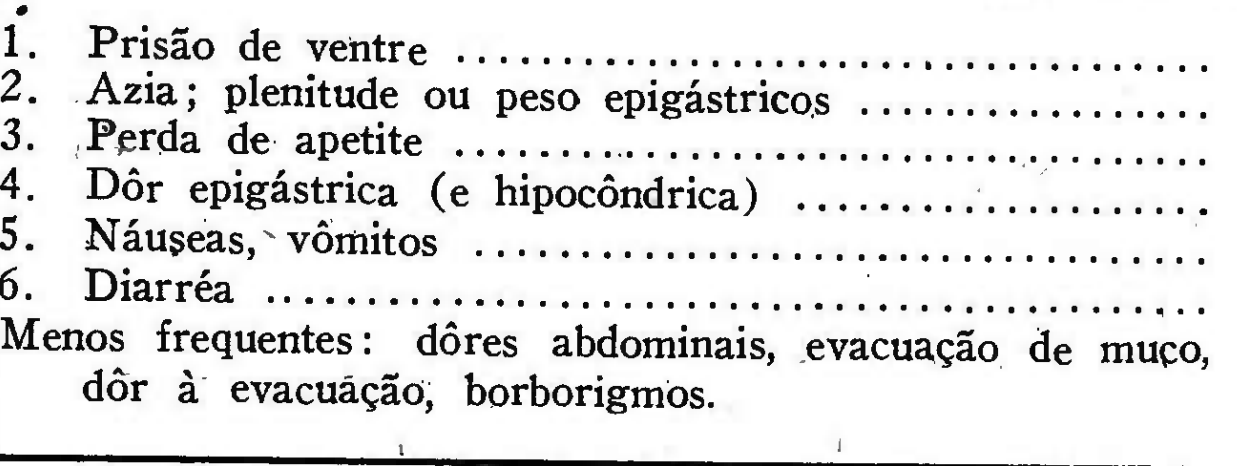 & $\begin{array}{rl}30 & \text { casos } \\
25 & " \\
22 & " \\
15 & " \\
12 & " \\
8 & "\end{array}$ \\
\hline
\end{tabular}

\section{QUADRO V-a \\ INSUFICIENCIA CARDÍACA 58 casos}

\begin{tabular}{|c|c|}
\hline Sem sintomas gastro-intestinais .... & 23 casos \\
\hline 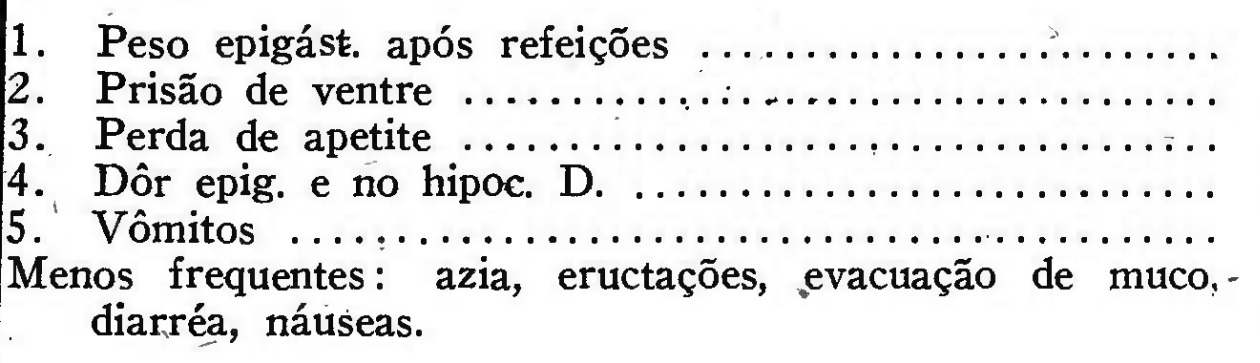 & $\begin{array}{l}16 \text { casos } \\
15 \\
13 \quad " \\
9 \\
8 "\end{array}$ \\
\hline 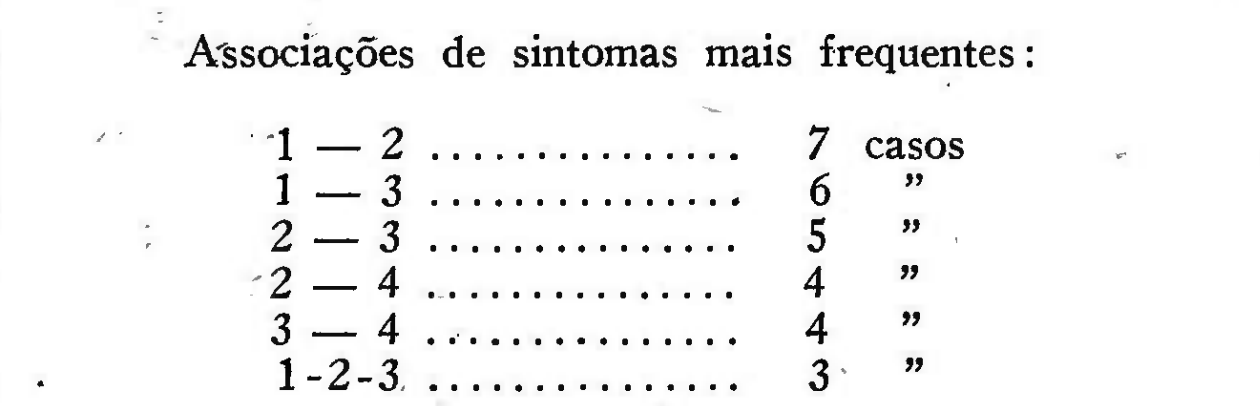 & $\cdot$ \\
\hline
\end{tabular}

Entre os grupos correspondentes áos dois períodos da idade adulta, a frequência de indivíduos que nắo apresentam sintomas digestivos é sensivelmente maior no grupo de indivíduos com mais de 40 anos. Neste grupo se apresentam menos frequentemente quasi todos os sintomas gastro-intestinais.

Móléstias de outros aparelhos - A frèquência de sintomas gàstro-intestinais nos casos de moléstias de outros aparelhos é demonstrada nos quadros seguintes. 


\section{QUADRO VI \\ MOLESTIAS DO FIGADO E DAS V. BILIARES 23 casos}

\begin{tabular}{|c|c|}
\hline Sem sintomas gastro-intestinais. & nenhum \\
\hline 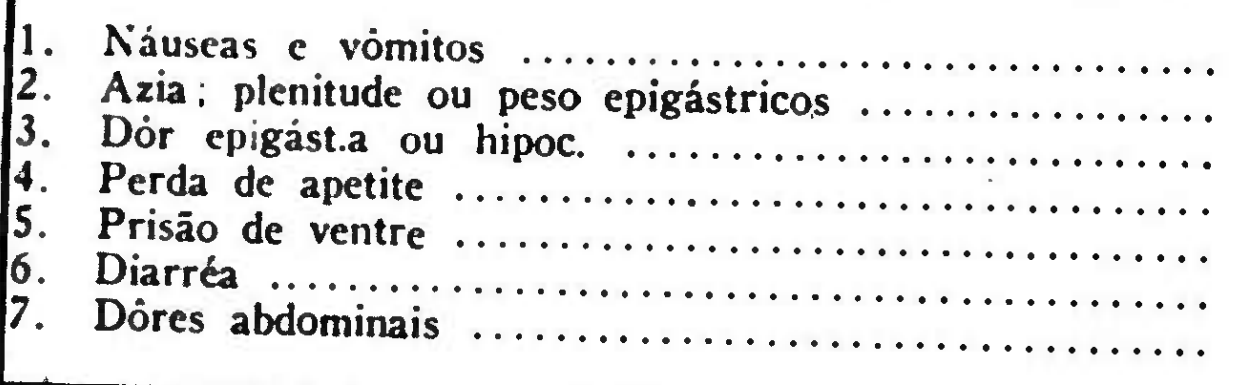 & $\begin{array}{l}14 \text { casos } \\
10 " \\
10 " \\
9 " \\
8 " \\
6 " \\
6 "\end{array}$ \\
\hline
\end{tabular}

Dos 81 casos de moléstias do anarelho respiratório, 7 apresentam associação com moléstias do estômago, dos intestinos, ou do fígado e vias biliares; ha ainda 10 casos em que coexistem afecções

As mole (Quadro IV).

observações estudo aparelho circulatório, verificadas em 105 das múiéstias do intestino em 11 casos, e com moléstias do aparelho res-
piratório em 10 .

Das 23 vezes que apareceram moléstias do fígado e das vias biliares (quadro VI), apenas em 5 coexistem moléstias de outros ór. gāos (estômago, intestino e aparelho respiratório).

Essas indicaçōes evidenciam como em todos esses casos a existência de afecções definidas do tubo gastro-intestinal se apresenta em desproporção com a alta frequência de casos com sintomas di-
gestivos.

\section{COMENTARIOS}

E consideravel a proporção dos doentes que se queixam de distúrbios que se traduzem por sintomas referidos, mais ou menos definidamente, ao tracto gastro-intestinal. Essa proporção, de 72 por cento, é muito superior à de indivíduos que no mesmo grupo apretestino ( 31,7 por cento).

Estariat por cento).

sintomas gastro-integtino, com grande frequência nos casos estudados, sória ou obrigatoriamente, "secundários", 1sto é que aparecem, acessente em outros órgãos.

do: sintomas enumatogênicas entre as moléstias principais e vários explicáveis. Mas é dignos nos quadros IV a VI são conhecidas ou são encontrados acompanhan nota que praticamente todos os sintomas tendo-se a impressão de que eles as moléstias dos vários aparelhos, 
condição mórbida fundamental em cada caso, como tambem a quaisquer causas de inferioridade funcional do próprio aparelho digestivo, presentes no grupo em estudo e tendentes a se manifestarem por sintomas diversos.

Uma causa dessa "inferioridade" do aparelho digestivo provavelmente se encontra em erros alimentares crônicos, mais que reconhecidos em nossas populações. Eles agiriam diretamente pelas condições de trabalho anti-fisiológicas em que colocam todo o aparelho digestivo; ou mediatamente, por mal definidos estados de carência, possibilidade aventada pelo Dr. A. de Ulhôa Cintra, na interessante proposição de problemas sobre "Estados de carência entre nós", que fez em recente conferência.

\section{LIVROS DE MEDICINA}

Consultem preços e condições com os seus colegas :

EDGARD SCHROEDER SAN JUAN

RUA BARÃO TATUHY, 120

3. ${ }^{\circ}$ ANO

5-1608

PAULO PEREIRA CORREIA

RUA HELVETIA, 760

3. ${ }^{\circ}$ ANO

$5-4238$ 